We describe our experience in the endoscopic management of migrated PS.

Methods A retrospective review of all ERCPs at our tertiary unit from January '13 -February '19 was performed to identify cases of migrated PS.

Results Twelve patients were identified; 10 received PS placement at other UK hospitals, 1 abroad and 1 at our unit. PS indications were prophylaxis of post-ERCP pancreatitis (PEP) in 9 and following pancreatic endotherapy in 3 (one for sphincter dysfunction, one for ductal stricture and one for cyst drainage). The migrated PS were $5 \mathrm{~cm}(\mathrm{n}=11)$ or $7 \mathrm{~cm}$ $(n=1)$ long with diameter of $3 \mathrm{Fr}(n=1), 5 \mathrm{Fr}(\mathrm{n}=10)$ and $7 \mathrm{Fr}$ $(n=1)$. Seven were straight and 5 single pigtail. 9 PS migrated after reported correct placement (of which 6 (67\%) were straight). Two were inadvertently deployed within the pancreatic duct (PD); in one case there was no information regarding placement. The site of PS migration was within the pancreatic head $(n=3)$, body $(n=3)$ and tail $(n=6)$.

After MDM review one patient was referred directly for surgery where a PD stricture precluded endoscopic access. Endoscopic removal was attempted in 11 patients and successful in 9 (82\%). 7 patients required two procedures. The median interval from stent placement to retrieval was 5 weeks (range 2-20). All patients had a previous pancreatic sphincterotomy. Techniques used for successful retrieval were: stent grabbers $(n=3)$, extraction balloon/stent grabbers $(n=1)$, snare/ stent grabbers $(n=2)$ and pancreatoscopy with SpyBite ${ }^{\text {TM }}$ forceps $(n=3)$. Endoscopic removal was unsuccessful in $2 / 11$ patients where pigtail stents had hooked into a side branch. In all cases a $5 \mathrm{~cm} / 5 \mathrm{Fr}$ prophylactic PS was placed post retrieval and 8 received rectal diclofenac (one contraindicated). Mild post-ERCP pancreatitis (PEP) occurred in one case.

Conclusions Endoscopic retrieval of proximally migrated PS was successful in $82 \%$ of patients. Migrated PS retrieval is challenging, and should only be attempted by endoscopists experienced in pancreatic endotherapy following case review within a specialist MDM. The mechanism of migration is unclear and spontaneous proximal migration of an appropriately placed single pigtail PS is unlikely to occur. Apparent migration in these cases most likely arises from incorrect deployment, potentially due to difficulty distinguishing the stent from the pushing device, emphasising the need for caution during PS placement. The optimal retrieval technique depends upon PS type, position, and PD anatomy. In our experience use of an extraction balloon alone was never successful. Failure of endoscopic retrieval was highest with pigtail stents and those $>2 \mathrm{~cm}$ from the papilla.

\section{PTH-022 ENDOSCOPIC RESECTION OF NON-AMPULLARY DUODENAL POLYPS: A RETROSPECTIVE SINGLE CENTRE EXPERIENCE}

Nishmi Gunasingam*, Mehul Patel, Shradda Gulati, Amyn Haji, Bu'Hussain Hayee. King's College Hospital NHS Foundation Trust, London, UK

\subsection{6/gutjnl-2019-BSGAbstracts.47}

Introduction Current literature estimates that complete endoscopic resection (ER) of duodenal adenomas can be achieved in $79-100 \%$ of cases, but complication rates are high and adenoma recurrence is encountered in up to $37 \%$ of cases (Basford \& Bhandari, 2012). We present our retrospective experience.
Methods Data from the electronic patient record was analysed for all patients who underwent duodenal polyp resection from June 2013 were included (excl. familial polyposis cases). Procedures were performed by either one of two endoscopists with experience in endoscopic resection. Accepted definitions of technical success, major adverse events and recurrence were used.

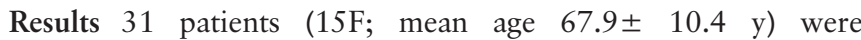
included. The mean polyp size was $38.8 \pm 23.6 \mathrm{~mm}$, with most ( $n=26$ ) located within D2. More than half were laterally spreading lesions $(n=16)$. The main method of resection was with piecemeal EMR $(n=24)$, with 5 removed by en-bloc EMR and 2 by ESD. Histology revealed tubular adenoma low grade dysplasia $(\mathrm{n}=12)$, tubulovillous adenoma with low grade dysplasia $(\mathrm{n}=11)$ and neuroendocrine tumour $(\mathrm{n}=$ 3 ). ER was successful in $28 / 31$ cases $(90.3 \%)$. Mean size in 3 incomplete resections was $93 \mathrm{~mm}$, with 1 patient referred for surgery, 1 repeat ER and 1 did not proceed due to a more pressing medical diagnosis.

$3 / 31$ had peri-procedural complications: endoscopicallytreated perforation in $2(6 \%)$ and minor bleeding in $1.2 / 31$ patients $(6 \%)$ experienced delayed bleeding, with one patient requiring a repeat OGD but no intervention and the other requiring transfusion of packed red blood cells and observation in hospital. There was no procedure related mortality. At time of writing 4 patients had not yet had surveillance OGD and to date 5 patients $(20.8 \%)$ had recurrence all treated endoscopically.

Conclusion ER of duodenal polyps is feasible and safe. Our single centre experience is on par with what is published in the literature in regards to technical success and adverse events. A prospective analysis would be of value to guide patient selection, optimal treatment and surveillance protocols.

\section{PTH-023 THE INCIDENCE \& INVESTIGATION OF IRON DEFICIENCY ANAEMIA IN PATIENTS PRESENTING WITH ACUTE CORONARY SYNDROME}

Paul Harrow*, Jessica Joseph, Rachael Hall, Sean Preston. Royal London Hospital, London, UK

\subsection{6/gutjnl-2019-BSGAbstracts.48}

Introduction Co-presentation with anaemia and acute coronary syndrome (ACS) is common. Anaemia is an independent risk factor for poor outcomes after ACS. Bleeding is the most common non-cardiac complication of ACS therapy. There are few studies existing on these patients. In this retrospective study we reported the incidence and characteristics of anaemia in patients presenting with NSTEMI $(n=55)$ or STEMI $(n=58)$ between 2015 and 2016 to a large tertiary centre.

Methods Patients were identified using an ICD10 code-based search. Data were collected from electronic patient notes and the pathology system. Endoscopy reports one year prior to and after the ACS were reviewed.

Results 45\% patients with NSTEMI and 28\% STEMI were anaemic at presentation with ACS. This was a microcytic anaemia in $36 \%$ patients. Iron saturations were tested or a recent result was available in $29 \%$ patients and ferritin was tested in $19 \%$ patients. These tests were more frequently performed in patients who had NSTEMI. When tested, iron saturations were low in $76 \%$ patients and ferritin was low in $29 \%$. 